\title{
Agricultura urbana y el rol de la planificación de las ciudades
}

Urban agriculture and the role of urban planning

Camilo Villagrán Oyarzún, Ken Yan Qiu Sun

\section{Filiación}

FAU - Universidad de Chile

Email: villagran.camilo@gmail.com,ken.yan.qs@gmail.com

\section{Resumen}

El presente artículo es parte de una investigación realizada el año 2013 la cual buscó indagar en los desafíos que presenta la inserción de la agricultura urbana en los Planes Reguladores Comunales en Santiago de Chile. Se presenta el estudio de dos casos en distintas comunas dentro del área Metropolitana, ya que se considera que su análisis permite inferir el estado de una situación general en relación a esta materia. Finalmente y como complemento a las conclusiones, se exponen algunas reflexiones generales respecto de las oportunidades que existen para su incorporación en la planificación urbana.

\section{Palabras Clave}

Agricultura urbana; planificación urbana; planes reguladores

\begin{abstract}
The following article is part of a research conducted in 2013 which sought to investigate the challenges of integration of urban agriculture in Communal Regulatory Plans in Santiago de Chile. Two case studies in different districts within the metropolitan area are displayed, as it is considered that its analysis allows to infer the state of a general situation in relation to this matter. Finally, as a complement to the findings, some general thoughts are presented, about the opportunities that exist for its incorporation into urban planning.
\end{abstract}

\section{Keywords}

Urban agriculture; urban planning; regulatory plans

\section{Sumario}

Introducción

1 Estudio de Casos

2 Síntesis de los Casos de Estudio

3 Conclusiones

4 Reflexiones

Bibliografía 


\section{Introducción}

Santiago de Chile es la mayor urbe del país y el crecimiento en extensión de su área urbana se ha traducido en una pérdida de suelo agrícola, entendiendo que dicho proceso implica el consumo de suelos rurales. Sin embargo, y de forma paradójica, para abastecer de alimentos a sus habitantes genera una gran demanda, que es suplida por áreas externas a sus límites, expandiendo aún más su área de influencia de manera virtual.

La producción agrícola de alimentos al interior de las áreas urbanas, es una actividad que en distintas partes del mundo cada día adquiere más fuerza, debido a los beneficios que aporta tanto a los habitantes de las ciudades como al equilibrio del medioambiente urbano, reduciendo el impacto de éste sobre el medio natural.

Cerca o dentro de las ciudades, la agricultura reduce la necesidad del transporte de la comida, cortando la dependencia de la ciudad en combustibles fósiles, y reduciendo emisiones de $\mathrm{CO} 2$ y otros contaminantes ${ }^{1}$.

La planificación urbana en este sentido puede incorporar aspectos ambientales y usos de recursos, como lo es la alimentación, formulando condiciones que permitan tener acceso a la tierra para poder realizar esta actividad.

De acuerdo a un catastro publicado en el año 2012 por la Red de Agricultura Urbana (RAU), existían 39 huertos urbanos en 20 comunas del Gran Santiago (valor que contabilizaba sólo lo registrado, por lo que eventualmente podían existir huertos no considerados en el estudio), tanto de carácter privado como municipal. Estas iniciativas de agricultura urbana han sido mayormente auto gestionadas por organizaciones interesadas en su implementación como una herramienta para alcanzar un desarrollo sustentable.

Integrar el desarrollo de la agricultura al contexto urbano de Santiago a través de la planificación, se traduce en que la ciudad se haga cargo (en parte) de la producción de uno de los recursos de los que más depende la población para subsistir, considerando los beneficios que esta actividad podría significar para el medioambiente urbano, en términos económicos, sociales y medioambientales.

En ese sentido, es importante comprender cómo funciona la planificación en las ciudades de nuestro país, especialmente en la capital, para constatar si es posible incorporar la agricultura desarrollada en este contexto al planeamiento de las comunas que la conforman.

\section{OBJETIVO PRINCIPAL}

La exploración realizada tuvo entonces como objetivo indagar en los desafíos que enfrenta la inserción de la agricultura urbana en los Planes Reguladores a través del estudio de casos puntuales en las comunas de La Reina (Huertos Municipales de la Aldea del Encuentro) y La Pintana (Huertos Familiares Mapuhue). El estudio se enfocó en la revisión de dichos instrumentos, entendiéndolos como factores relevantes para la promoción de la agricultura en la ciudad. Esto fue complementado con entrevistas a diversos actores involucrados directamente en el tema.

\section{HIPÓTESIS}

Se sostiene como hipótesis que el mayor desafío para la inserción de la agricultura en la planificación urbana es su incorporación a la agenda política, ya que existe una falta de visión y proyección a futuro por parte del Estado en el ámbito alimentario y su impacto medioambiental. Debido a la falta de reconocimiento del tema por parte de los gobiernos, la

${ }^{1}$ WWF 
agricultura es una actividad considerada como ajena al ámbito de la ciudad según el marco normativo de la planificación urbana. Actualmente, la producción agraria de alimentos se remite al sistema rural y, por lo tanto, se excluye de las estrategias de desarrollo urbano.

Si bien su reconocimiento en la planificación no es el único factor que influye en su desarrollo, se estima que el hecho de que actualmente la agricultura urbana no se considere, ha incidido significativamente en la escasa proliferación de las iniciativas en Santiago. Los casos estudiados, los Huertos Municipales de la Aldea del Encuentro en la Reina y los Huertos Familiares Mapuhue de La Pintana, ambos de larga data, han logrado mantenerse dentro de un marco normativo ambiguo, por lo que resulta complejo pensar en la posibilidad de replicar dichas experiencias amparándose en los Planes Reguladores Comunales.

\section{SOBRE LA PLANIFICACIÓN URBANA EN SANTIAGO DE CHILE}

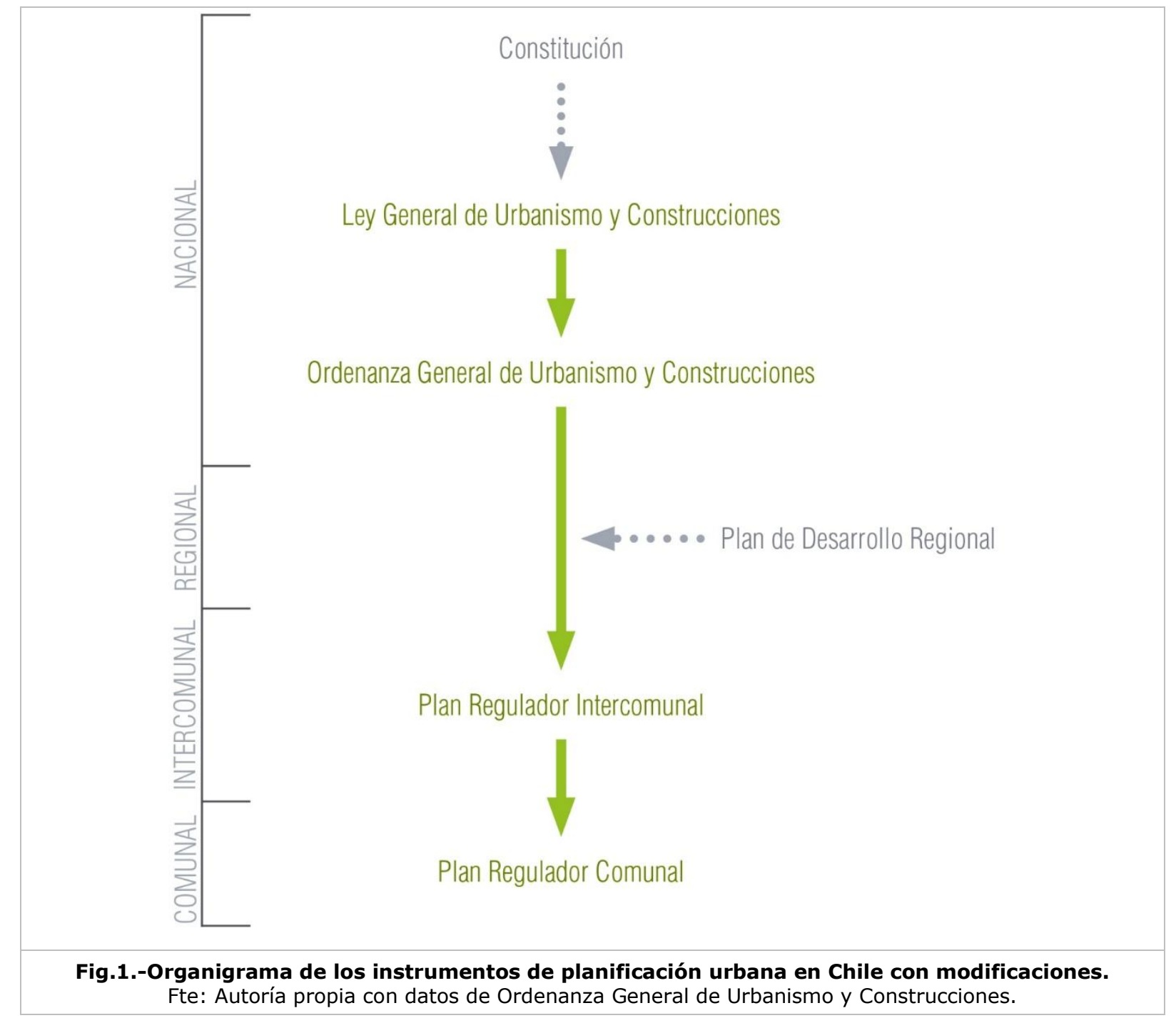


La planificación urbana chilena se encuentra regulada en diferentes instrumentos, dispuestos en orden jerárquico lineal donde "las normas inferiores deben ser acordes con las superiores y en ningún caso pueden transgredir lo prescrito por una norma de mayor rango"2.

En este esquema de instrumentos cobran especial relevancia la Ley General de Urbanismo y Construcciones (en adelante LGUC) y la Ordenanza General de Urbanismo y Construcciones (en adelante OGUC), ya que son aquellos con mayor relevancia en la escala de los instrumentos normativos del país. La LGUC es un instrumento de carácter general que toma la orientación señalada por la política chilena de desarrollo urbano, estableciendo, entre otros aspectos, los principios que regulan la materia. Por su parte, la OGUC, de acuerdo a lo expresado por el artículo 2 de la LGUC:

"Contiene las disposiciones reglamentarias de esta ley y regula el procedimiento administrativo, el proceso de planificación urbana, urbanización y construcción, y los estándares técnicos de diseño y construcción exigibles en los dos últimos"'3.

Además de las normas anteriores, se encuentran los Planes Reguladores, los que de acuerdo a la división político-administrativa del país se clasifican en: regionales, intercomunales o metropolitanos, y comunales. Para efectos de la presente investigación, cabe señalar que los Planes Reguladores Comunales son aquellos instrumentos que establecen los lineamientos para la planificación física del área comunal, lo que se refiere, entre otros, a los usos de suelo, a la jerarquización de la estructura vial, a la fijación de límites urbanos, y a las normas de edificación (coeficientes de constructibilidad, ocupación de suelo, etc.) siendo entonces los de índole más acotada y definida. Su especificidad determina la gran relevancia que tienen en el desarrollo físico del territorio urbano, razón por la cual se decidió avocar su estudio directo para la recopilación de información de cada caso a estudiar.

\section{Estudio de Casos}

\subsection{HUERTOS FAMILIARES MAPUHUE, LA PINTANA.}

\subsubsection{Revisión de la Normativa}

La Pintana es una de las trece comunas de la región metropolitana que se originó debido al reordenamiento del Gran Santiago en el año 1981. Anteriormente, el sector era parte de la comuna de La Granja, por lo que se rige, hasta la fecha, por el Plan Regulador de la Granja y el Plan Regulador Metropolitano de Santiago (en adelante PRMS).

Desde el año 2008, existe un proyecto impulsado por la Secretaría de Planificación para la creación del llamado Plan Regulador Comunal de La Pintana (en adelante PRCLP), el cual se encuentra a la espera de ser aprobado y será objeto de estudio en esta investigación.

\footnotetext{
2 FERNANDEZ Y HOLMES, 2012; 94

${ }^{3}$ LGUC
} 
Revista de Urbanismo N²9 - Diciembre de 2013

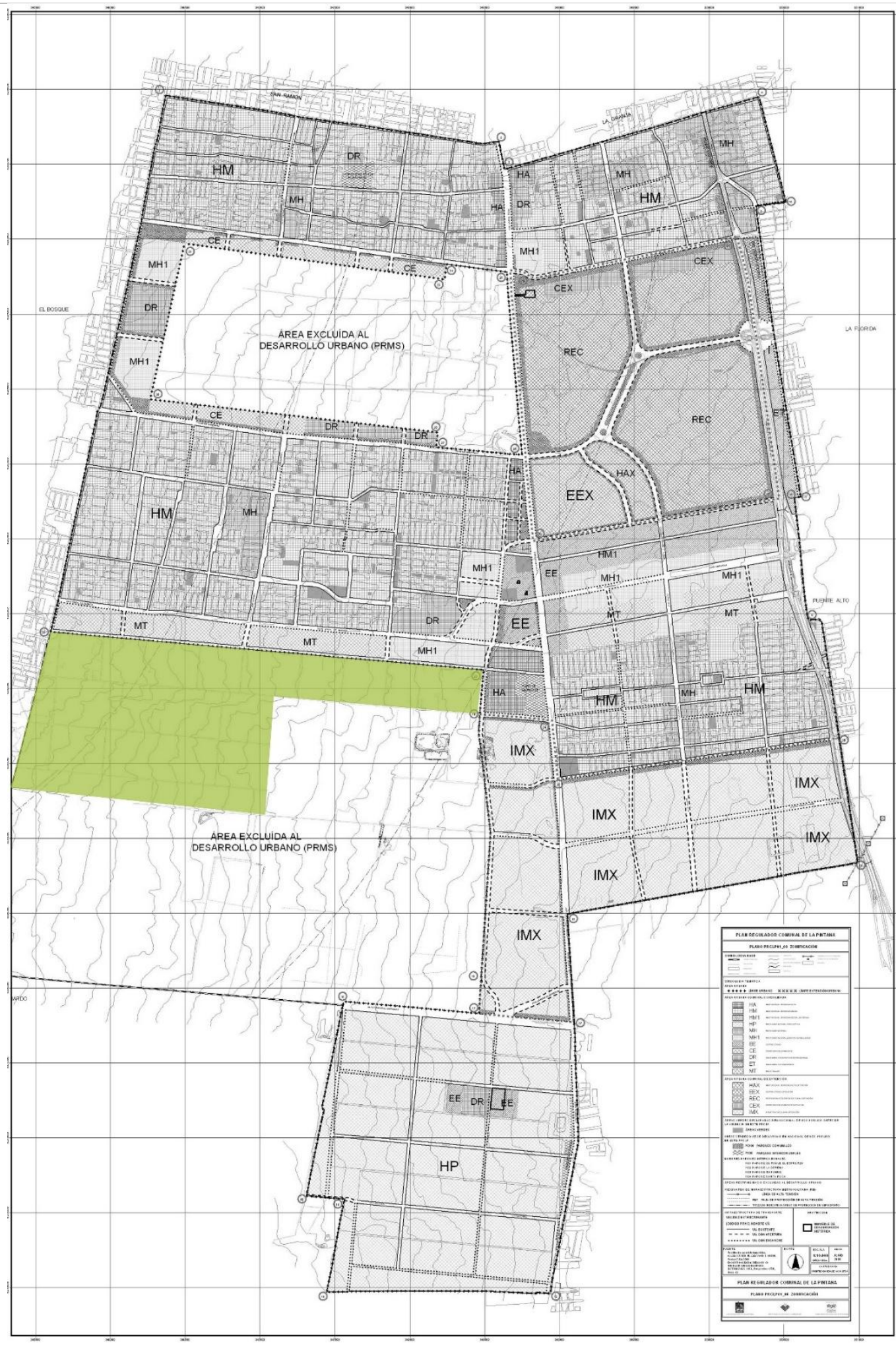

Fig.2.- Proyecto Plan Regulador Comunal La Pintana, 2008.

Fte: I. MUNICIPALIDAD DE LA PINTANA. Proyecto Plan Regulador Comunal [modificado por los autores] 
En el proyecto de PRCLP los Huertos Familiares Mapuhue están ubicados en un Área de Interés Agropecuario Exclusivo, la que corresponde a una zona excluida del desarrollo urbano y que, por lo tanto, no se rige por lo expresado en el PRCLP sino por el PRMS encontrándose bajo el control del Ministerio de Agricultura. El PRMS define esta área como aquellas "con uso agropecuario cuyo suelo y capacidad de uso agrícola debe ser preservado". Sus características, según el artículo 8.3.2. y 8.3.2.1. del PRMS son:

- Subdivisión predial mínima de 4 hectáreas. Con una vivienda por predio. Además se podrá edificar en el mismo predio una vivienda para un cuidador (bajo las condiciones de vivienda social).

- Si el Municipio lo requiere, se pueden emplazar conjuntos de viviendas sociales para campesinos en terrenos con un mínimo de $100 \mathrm{~m}^{2}$ de superficie, cumpliendo ciertas condiciones.

- Se autoriza la instalación de agroindustrias que procesen productos frescos.

- Se permiten Desarrollos Urbanos Condicionados.

- Se permiten Desarrollos Industriales y/o Empresariales Condicionados.

Dentro del proyecto del Plan Regulador se pretende integrar esta zona al área urbana en calidad de "actividad agrícola urbana", pero el proyecto de modificación al PRMS (PRMS100, cuya actualización no está aprobada) no considera esta propuesta y, al contrario, expandía el límite urbano, cambiando el uso de suelo del sector a Habitacional Mixto, afectando por consiguiente su identidad como sector agrícola. Lo anterior deja en manifiesto la pugna existente entre las disposiciones del PRMS y del PRCLP, las cuales no han sido resueltas hasta el día de hoy.

Además, en la memoria del proyecto de PRCLP se presenta otra idea sobre la producción agrícola en la comuna: el uso de zonas aluviales como potenciales áreas cultivables, lo cual no se encuentra explícitamente en el plan regulador. También existen iniciativas de conservación ecológica y cultural, específicamente en el área de La Platina (de 190 hectáreas), pero no se menciona ningún uso relacionado con la agricultura como actividad permitida.

A pesar de que el proyecto de PRCLP presenta intenciones sobre la integración de la agricultura a la zona urbana, éstas no son concretadas en el instrumento final. En el caso de las Huertas Familiares Mapuhue, se busca integrar el sector a la zona urbana manteniendo sus características, pero no es clara la manera en que se pretende lograr este objetivo, por lo que al aprobarse el proyecto de plan regulador es probable que se mantenga la incertidumbre y la tensión en el sector.

Una posible vía para mantener las características normativas de Mapuhue y permitir su inclusión al Plan Regulador es su transformación en Área de Interés Silvoagropecuario Mixto (en adelante ISAM), la cual, según el artículo 8.3.2.2. del PRMS, presenta características adicionales al Área de Interés Silvoagropecuario Exclusivo según las solicitudes 0 requerimientos del proyecto, los cuales pueden ser modificados conforme a los objetivos del sector.

En las áreas de ISAM es posible proponer, bajo una serie de estudios aprobados, la incorporación de áreas en las que es posible desarrollar loteos de parcelas agroresidenciales al plan regulador comunal, bajo las siguientes condiciones: 
- Contar con urbanización completa en términos de infraestructura sanitaria, energética y de pavimentación.

- Dar cumplimiento a lo establecido en la OGUC respecto de cesiones de terreno para áreas verdes, equipamiento y circulación.

- Superficie predial mínima de 0,5 hectáreas.

- Coeficiente de constructibilidad máxima equivalente a 0,1.

- Además de la vivienda del propietario, es posible construir una vivienda para cuidador, similar a lo señalado respecto de las Áreas de Interés Silvoagropecuario Exclusivo.

En el PRMS se presentan trece áreas de ISAM, en las cuales, además de repetirse las condiciones mencionadas con relación a la vivienda, se presentan distintos usos y actividades (permitidas y prohibidas) definidas específicamente para cada sector, tales como la inclusión de industria, comercio y la prohibición de viviendas.

Como fue expresado, el área de ISAM puede integrarse al plan regulador, pudiendo ser intervenida por los planificadores comunales; sin embargo, es una herramienta con poco grado de detalle, pues se manifiesta como una gran área homogénea que admite diversos usos (por ejemplo: la agricultura), a diferencia de aquellas otras zonas con características específicas que permiten elaborar un sector planificado con normas claras y estructuradas. Además, cabe señalar la rigidez que presentan las áreas de ISAM con respecto al ámbito residencial, pues su objetivo es mantener un sector con baja densidad poblacional.

\subsubsection{Entrevistas del caso de estudio}

Cecilia Bahamonde, secretaria de la Cooperativa de Huertos Mapuhue, reconoce que parte de la villa mantiene un uso silvoagropecuario. Está al tanto de que el área norte comprendida entre calles Tucapel y lo Blanco posee un uso mixto (ya que esa franja del área de Mapuhue sí está integrada a la normativa urbana. Manifiesta, además, que existe un conflicto con los procesos que han significado la introducción de elementos relativos al ámbito urbano, como sucede con la reciente pavimentación de caminos, la instalación de talleres de desarmadurías, etc.

Producto de la parcelación y existencia de nuevos propietarios, hoy en día la cooperativa tiene un rol poco preponderante en el sector de Mapuhue. Cada productor comercializa sus cultivos independientemente, los que preferentemente se destinan a las ferias libres de la zona.

Ella señala preferir que las condiciones actuales se conserven, ya que a su parecer, mantenerse como un área silvoagropecuaria les permite proteger las características que los convierten en un pulmón verde de Santiago. A su vez, reconoce que en la comuna se han ido perdiendo zonas de producción agrícola y que, por lo mismo, las asociaciones de huerteros existentes están luchando para que sus propiedades sigan siendo áreas de cultivo, oponiéndose a las presiones del PRMS.

Graciela Ramos, asesora urbana del municipio de la Pintana, comenta que, en el caso del área de Mapuhue, parte de ésta se encuentra fuera de la zona de acción del plan regulador comunal, ya que en este momento mantiene el estatus de zona de protección silvoagropecuaria, por lo que como Secretaría de Planificación Comunal (en adelante SECPLAC) no tienen suficiente injerencia en cuanto a lo que sucede en dicho territorio. Reconoce estar al tanto de las presiones que existen por parte del PRMS para catalogar este sector como una potencial área urbana condicionada factible de ser incorporada al límite urbano pero que, ello, es una decisión que toma el gobierno regional, no el SECPLAC.

Personalmente, manifiesta que se debiese propiciar la agricultura urbana, por ejemplo, a través del uso de los terrenos eriazos de propiedad fiscal, sin embargo, hace hincapié en que más allá 
de ser un asunto que concierne a la planificación, se trata de un problema de gestión. Señala a su vez que, en el caso concreto de Mapuhue, sería positivo generar un plan al respecto, pero esto resulta ser un escenario complejo, ya que debiese formar parte de la política de desarrollo comunal.

Considera sumamente restringido que, en materia de planificación, las variables normadas por los Planes Reguladores deban restringirse a lo dispuesto por la OGUC. En efecto, el concepto de agricultura no aparece en ninguna parte como un uso permitido, no obstante, dice que los planificadores podrían manejar las variables que existen para posibilitar el desarrollo de la agricultura en la ciudad.

Finalmente, comenta que la política de desarrollo urbano no puede desentenderse del modelo socioeconómico imperante. Si bien en definitiva, es el mercado el que manda, el Estado no debería desentenderse de su labor, ya que está en juego el bien común de la población. Concluye que la agricultura dota de externalidades positivas al medio ambiente urbano, por lo que debiese defenderse.

\subsection{HUERTOS MUNICIPALES ALDEA DEL ENCUENTRO, LA REINA}

\subsubsection{Revisión de la Normativa}

La comuna de la Reina se originó el año 1963 luego de desprenderse administrativamente de la comuna de Ñuñoa. Es una de las 5 comunas de la provincia de Santiago que se encuentra en el piedemonte andino, por lo que parte de su territorio posee grandes pendientes que han servido para mantener una baja densidad y abundante arborización.

El Plan Regulador vigente data del año 2001 y regula las normas de edificación, los usos de suelo y las actividades permitidas en el territorio comunal, entre otras, siendo aplicable tanto a las áreas definidas como urbana y urbanizable.

En el Plan Regulador Comunal, expresado en el respectivo plano de zonificación y ordenanza, el área en la cual se emplazan las Huertas de la Aldea del Encuentro está definida como un Polo de Desarrollo de Equipamiento Comunal (PC-5 Avenida Larraín - Municipalidad), zona para la cual rigen las normas específicas determinadas para la ZONA $P$ (Polos de Desarrollo de Equipamiento Comunal y Vecinal).

Las características definidas para esta zona son las siguientes:

- Vivienda y Equipamiento de escala comunal son permitidos como usos de suelo.

- En el caso de las viviendas se exige una superficie predial mínima de 3500m2.

- Tabla de normas específicas para vivienda 


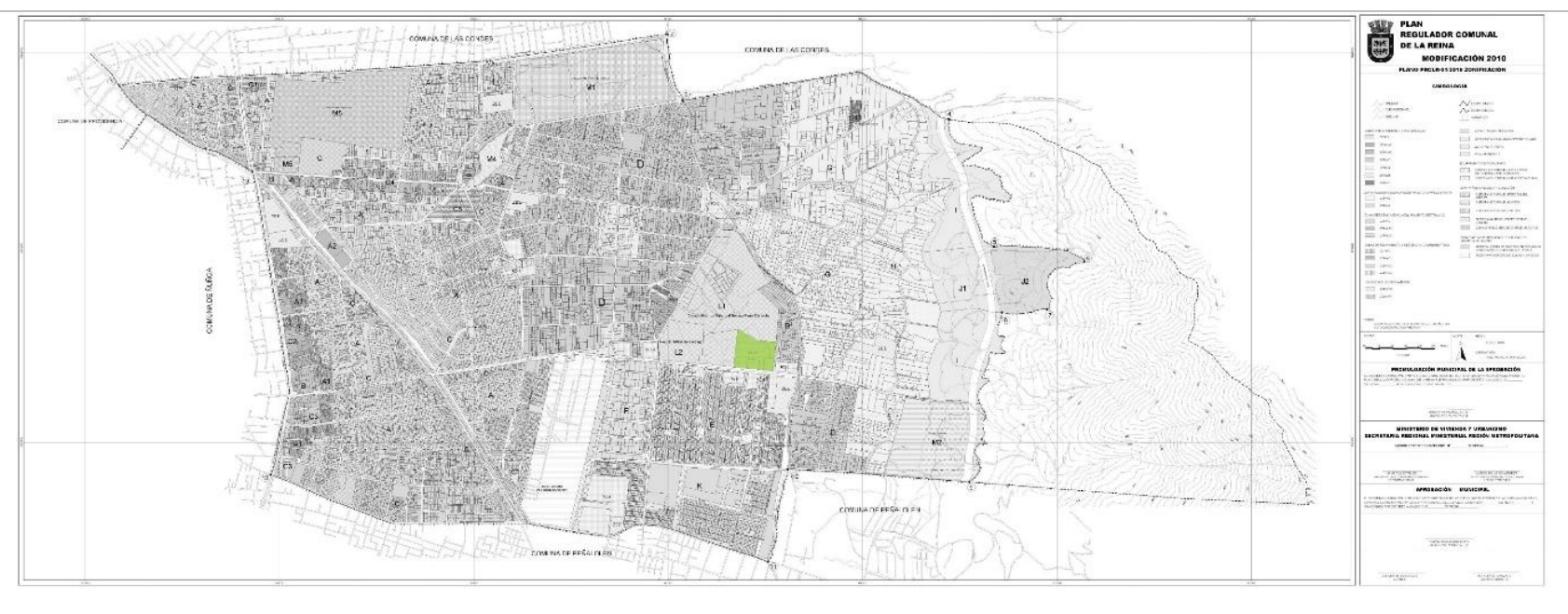

Fig.3.- Plan Regulador Comunal La Reina, 2001

Fte: I. MUNICIPALIDAD DE LA REINA. Plano de Zonificación [modificado por los autores]

En el sector definido como PC-5, además de la Aldea del Encuentro, se encuentran las dependencias de la municipalidad, el Centro Nacional del Medio ambiente, un colegio, entre otros, situación que da cuenta de la vocación pública que ha adquirido dicho polo. De acuerdo a lo especificado por el plan regulador, se definen otras cuatro zonas como polos de equipamiento comunal (Zonas P), sin embargo, solo en la zona PC-5 es posible encontrar agricultura urbana, ya que éstos han tendido a usos comerciales y de servicios, adquiriendo otros caracteres.

Respecto a las huertas municipales, hay que considerar que éstas se emplazan de forma adyacente a una edificación que se constituye como equipamiento de escala comunal, la que alberga las dependencias de la Corporación Aldea del Encuentro. Sin embargo, dentro de todas las actividades que se permiten desarrollar en la zona PC-5, no aparece expresamente el de huertas y/o cultivos. Esto da cuenta de que si bien existe una regulación en términos de la construcción, ello no acontece en el caso de los huertos, por lo que se hace evidente la existencia de un vacío en este aspecto, por lo que es posible que los huertos se consideren como parte de las áreas verdes del complejo de la Corporación.

Una revisión del plano de zonificación del territorio comunal da cuenta de la existencia de varias áreas verdes y recreacionales, tanto de escala regional y metropolitana como de escala comunal y vecinal ${ }^{4}$, evidencia de la vocación de parque con la que se reconoce a la comuna.

\section{SUBZONA M1 PARQUE INTERCOMUNAL ORIENTE}

SUBZONA M2 PARQUE MUNICIPAL

SUBZONA M3 AVENIDAS PARQUE

SUBZONA M4 AREAS VERDES DE NIVEL COMUNAL

\section{SUBZONA M5 AREAS VERDES COMPLEMENTARIAS}

Además existe un área definida como SUBZONA 32 excluida del desarrollo urbano, por ser un área de protección ecológica ${ }^{5}$. Para todas estas zonas de áreas verdes, ya sean parques,

${ }^{4}$ I. MUNICIPALIDAD DE LA REINA. ref. 124 
plazas, jardines botánicos, jardines de plantas se permiten usos compatibles dependiendo de su escala pudiendo ser científicos, culturales, educativos, recreacionales, deportivos y turísticos, considerando las instalaciones y/o edificaciones mínimas e indispensables para su habilitación. Sin embargo, en la ordenanza no se menciona explícitamente ningún uso relativo a la agricultura como actividad permitida y/o compatible con áreas verdes. Las subzonas definidas como áreas verdes en el instrumento son:

- CULTURA

- Jardines botánicos

- AREAS VERDES

- E. metropolitana: parques nacionales, santuarios de la naturaleza, grandes parques

- E. comunal: parques, plazas

- E. vecinal: plazas, jardines.

- SERVICIOS ARTESANALES

- E. vecinal: jardines de plantas.

De acuerdo a los antecedentes recopilados, las huertas estarían "a salvo" porque se desarrollan en un terreno municipal. Sin embargo, en el Plan Regulador Comunal, no se estipula nada respecto a la producción agrícola de baja escala. Probablemente esta protección se da porque, hasta ahora, no han existido conflictos entre la Corporación a cargo de la iniciativa y las distintas administraciones municipales que se han sucedido desde el año 2001.

\subsubsection{Entrevistas del caso de estudio}

La coordinadora de los huertos, Julia Franco, expresa que la iniciativa no tiene por objeto generar un negocio en términos económicos, sino que busca, por un lado, ser una alternativa que permita a las personas tener una alimentación más saludable, y por otro lado, pretende destacar todos los aspectos positivos asociados a la agricultura en el ámbito urbano, tanto para la gente involucrada (en lo relativo a las relaciones sociales y a la salud física y sicológica) como en lo que respecta a los beneficios medioambientales.

Estima relevante que el municipio cuente con una ordenanza que permita a los vecinos poder cultivar en espacios públicos, como por ejemplo en las platabandas de las calles, situación que hace tiempo intenta gestionar. Sumado a esto, le interesan dos potenciales alternativas para promover los cultivos agrícolas en el contexto urbano; por un lado, la posibilidad de utilizar terrenos baldíos en la ciudad para hacer agricultura, y por otro, el cambio en el concepto de área verde, el que hasta ahora, se limita a considerar la vegetación como un ornamento.

Plantea a su vez que, como huerteros urbanos, no cuentan con apoyo financiero por del Estado, y que, por determinadas condiciones, tampoco pueden acceder a fondos que les permitan mejorar sus cultivos. En este sentido, estima que el MINAGRI debiese contar con un departamento de agricultura urbana.

Fundamentalmente, según su experiencia, el poco desarrollo o preocupación por parte de las instituciones públicas, se debe, principalmente, a un desconocimiento o falta de claridad en cuanto a lo que concierne a la agricultura urbana, y que en este sentido, somos el país más atrasado de toda América Latina.

${ }^{5}$ I. MUNICIPALIDAD DE LA REINA. ref. 125 


\section{REVISTA DE \\ URBANISMO}

ISSN 0717-5051

http://revistaurbanismo.uchile.cl
Revista de Urbanismo N²9 - Diciembre de 2013 Departamento de Urbanismo - FAU - Universidad de Chile

David Belmar, asesor urbano de la municipalidad, comenta que la Reina posee un origen asociado a lo agrícola, destacándola en la actualidad por ser una comuna "verde" debido a la abundante forestación y existencia de parcelas de agrado, reconociendo un potencial respecto al tema, tanto en lo urbano como en lo social.

En el caso de la Aldea del Encuentro estima que si bien se trata de una iniciativa destacable y que sería positivo que ideas se replicara, lo acotado de la misma no tiene implicancias significativas en el territorio comunal que haga necesario generar políticas municipales al respecto. A sí mismo, considera que otras acciones de agricultura urbana, al igual que la Aldea del Encuentro, en términos de normativa y de usos potenciales podrían incorporarse ya sea a los Polos Comunales de equipamiento o a determinadas áreas residenciales pero que, finalmente, debido a las dinámicas culturales y a las lógicas de mercado imperantes, éste sería un escenario complejo de concretar que quizá no se corresponde con la dirección en la que se orienta el desarrollo de la ciudad ni con las necesidades actuales de los habitantes.

Por último, comenta que, como esto es un tema reciente, tal vez sería necesario contar con más estudios que la relevancia de la incorporación de la agricultura al ámbito urbano

\section{Síntesis de los Casos de Estudio}

\begin{tabular}{|c|c|c|c|}
\hline & & HUERTOS MUNICIPALES, LA REINA, CHILE & HUERTOS FAMILIARES MAPUHUE, LA PINTANA, CHILE \\
\hline \multirow{4}{*}{$\begin{array}{l}\text { FACTORES DE } \\
\text { NORMATIVA } \\
\text { URBANA }\end{array}$} & Nombre Instrumento & Plan Regulador Comunal de La Reina, 2001 & $\begin{array}{l}\text { Plan Regulador Comunal de La Pintana, } 2008 \text { (proyecto no } \\
\text { aprobado) }\end{array}$ \\
\hline & Escala de planificación & Comunal & Comunal \\
\hline & $\begin{array}{l}\text { Zonificación relacionada a la } \\
\text { agricultura }\end{array}$ & $\begin{array}{l}\text { Ninguna relacionada a la agricultura. El terreno se } \\
\text { emplaza en la zona PC- } 5 \text { como Polo Comunal, que no } \\
\text { permite nada relacionado a la agricultura o el cultivo }\end{array}$ & $\begin{array}{l}\text { Ninguna relacionada a la agricultura. El terreno se emplaza } \\
\text { en un Área de Interés Silvoagropecuario Exclusivo, por lo que } \\
\text { queda excluída al desarrollo urbano y fuera de la jurisdicción } \\
\text { del Plan Regulador Comunal }\end{array}$ \\
\hline & Actividades permitidas & $\begin{array}{l}\text { Ninguna relacionada a la agricultura. El terreno don- } \\
\text { de se emplazan los huertos municipales de La Reina es } \\
\text { propiedad de la Municipalidad, por lo que permiten el } \\
\text { cultivo sin estar normado en el Plan Regulador }\end{array}$ & $\begin{array}{l}\text { Ninguna relacionada a la agricultura. Al ser un Área de } \\
\text { Interés Silvoagropecuario, es permitido el cultivo y la actividad } \\
\text { agroindustrial, pero el terreno se convierte en una zona rural, } \\
\text { ajena al resto de la comuna }\end{array}$ \\
\hline
\end{tabular}

Tabla 1.- Tabla de indicadores según el estudio de los instrumentos normativos Fte: Elaboración propia

A través de la presente revisión, fue posible corroborar que en las comunas estudiadas la agricultura existe como una actividad desarrollada por iniciativas específicas impulsadas por agrupaciones ciudadanas interesadas en hacer crecer las iniciativas existentes, sin embargo, se desarrollan en contextos muy distintos.

Los Huertos Municipales de la Aldea del Encuentro en La Reina forman parte de un complejo mayor que integra diversos equipamientos comunales, por la cual están muy integrados al área en la que se emplazan, definida por el Plan Regulador Comunal como un Polo de Desarrollo de Equipamiento, uso que se regula mediante la normativa urbana, pero que no consagra expresamente la actividad agrícola. Respecto al desarrollo de la agricultura urbana en el área comunal, los huertos representan una iniciativa puntual y acotada, que no ha tenido mucha incidencia en el territorio.

En la Pintana, los Huertos Familiares Mapuhue ocupan una extensa área del territorio comunal en la que se pudo comprobar la existencia de un vínculo estrecho entre residencia y actividad agrícola. A pesar de que en este caso la agricultura si está considerada por la normativa, esta zona se regula bajo criterios rurales, por lo que está totalmente desasociada del resto del territorio comunal, (definido mayormente como urbano. En la actualidad, el área de Mapuhue se integra al PRMS como una zona de interés silvoagropecuario mixto. No obstante, ocurriera un cambio en la regulación la situación no variaría mucho ya que por las variables que 
condicionarían dicha zona evidentemente ésta seguiría siendo una "mancha" rural dentro del límite urbano más que una zona urbana.

\section{Conclusiones}

La visión del Estado en relación a la producción agrícola se refleja en los instrumentos vigentes sobre la planificación territorial en Chile, ya que sus restricciones impiden que se implemente como actividad urbana en los Planes Reguladores, pues éstos no pueden agregar nada que no se contemple en documentos legislativos jerárquicamente superiores (LGUC y OGUC), los cuales circunscriben la agricultura como una actividad exclusivamente rural, evidenciando con ello un enfoque limitado de los instrumentos en este sentido. Una modificación en éstos implica un cambio en el pensamiento tanto de los planificadores como de las autoridades en Chile, lo que podría devenir en una sucesión de oportunidades favorables para la inserción de la agricultura urbana a los planes reguladores comunales.

Julia Franco, coordinadora y fundadora de los huertos municipales de La Reina, afirma que existe un desconocimiento general sobre el tema, siendo esto una de las mayores limitaciones para la inserción de la agricultura en la zona urbana. La falta de conocimiento incide directamente en la inexistencia de políticas gubernamentales (en toda la escala jerárquica) referentes a la agricultura urbana. Este hecho viene a demostrar la divergencia entre el pensamiento de los actores estatales y aquellos que conciben la agricultura urbana como una iniciativa beneficiosa para la ciudad y sus habitantes.

La confianza de las autoridades en el actual modelo globalizado de producción alimenticia, se ve reflejada en las políticas y lineamientos de la planificación. El fortalecimiento de la agroindustria y la red alimenticia globalizada es uno de los objetivos del Ministerio de Agricultura, el cual busca emplazar a Chile en el mundo como una potencia agroexportadora. Si bien, la producción agrícola intensiva en las zonas rurales es esencial para satisfacer la demanda alimenticia, la agricultura urbana no sólo contribuye a la seguridad alimentaria, sino que también refuerza los ámbitos sociales, económicos y ambientales. Así, ésta puede funcionar como una actividad complementaria a la agricultura tradicional y a la agroindustria rural, generando un sistema alimentario integral, disminuyendo el impacto medioambiental de las ciudades y produciendo externalidades positivas para la población y la economía local.

La planificación requiere de una estrategia coherente y unificadora, tanto a nivel nacional, regional e intercomunal, pero, según informes de la OCDE, ello resulta de difícil aplicación en Chile ya que esta actividad se basa en proyectos y no en estrategias a largo plazo, de manera que se torna complejo llevar a cabo iniciativas de carácter proactivo sin contar con objetivos bien establecidos.

Solo una vez que el Estado chileno tome conciencia real del alcance de la agricultura urbana como iniciativa favorable para un desarrollo sostenible, inserte el tema en la agenda política, y defina una perspectiva estratégica que favorezca el trabajo en conjunto, podrá abrirse una puerta para la inserción de la agricultura urbana en los instrumentos de planificación de Chile, la que podrá funcionar como una poderosa herramienta para tratar de alcanzar un desarrollo urbano sustentable.

\section{Reflexiones}

Integrar la agricultura a la normativa urbana responde a la intención de recurrir a ella como una herramienta con la cual poder contar para planificar en el ámbito urbano. Existen oportunidades que permitirían su incorporación, tanto en las zonas urbanas más consolidadas 
como en las áreas periurbanas, lo que facultaría a generar una mayor integración entre el área urbana y el área rural.

Las "zonas agrícolas periurbanas pueden ser incluidas en los planes de desarrollo de la ciudad como parte de cinturones verdes o corredores verdes con el fin de evitar el desarrollo descontrolado y la destrucción de los suelos"6.

Además, la inclusión simbiótica de la agricultura a la planificación, contribuiría a desarrollar zonas de amortiguamiento de las áreas urbanas más consolidadas, a la vez de servir al mejor direccionamiento del desarrollo de las áreas rurales.

Una de las tantas formas en que se podría incorporar la agricultura al ámbito urbano, que ha sido señalada por algunos entrevistados y que se evidencia en los casos internacionales, es la generación de un cambio en el concepto de área verde para integrar las zonas agrícolas a la ciudad, pues hasta el momento se encuentran relegadas silvoagropecuarias.

En nuestro país la normativa urbana se restringe a concebir la vegetación con fines puramente ornamentales, a pesar de que los cultivos agrícolas aportan los mismos beneficios ambientales en cuanto a captura de $\mathrm{CO} 2$ y ayudan al embellecimiento del paisaje urbano, entre otras cosas, e incluso, entregan la posibilidad de ser aprovechados como alimentos. Es preciso considerar que no solo las hortalizas, sino también, hierbas rastreras y flores comestibles junto a los árboles frutales pueden aportar en ese sentido. A su vez, asociando este concepto a los espacios públicos se podrían complementar parques y plazas, recuperar los que se encuentren deteriorados, así como constituir una alternativa para nuevos espacios de uso público.

"La introducción de huertos al sistema verde supone una fusión entre las nuevas actividades generadas por los huertos y las funciones ya consolidadas de las áreas verdes; combinando cultivos, jardines y mercados con campos de juegos, plazas y bulevares. Así, los parques podrían convertirse en centros comunitarios de reciclaje y compostaje, pequeños mercados, que beneficien a la comunidad"'.

Otra alternativa para insertar la agricultura urbana es por medio de la utilización, al menos temporalmente, de sitios eriazos o terrenos vacantes en el interior de la ciudad, ya sean de carácter público o privado. Los sitios eriazos, áreas deterioradas ambiental y paisajísticamente, muchas veces representan focos de inseguridad para la población además de que en términos ambientales representan islas de calor para la ciudad perjudicando, por consiguiente, el clima urbano $^{8}$. De esta forma, la agricultura se torna en una opción para prevenir la delincuencia y la acumulación de desechos ${ }^{9}$. Ahora bien, para implementar estas opciones las municipalidades tendrían que autorizar el uso de terrenos vacantes en zonas urbanas para que grupos organizados los utilizaran en la producción de alimentos.

Podría plantearse a su vez, la posibilidad de incorporarla como parte de equipamientos e infraestructuras de uso públicos, tales como hospitales, escuelas, etc. así como en proyectos de viviendas sociales y económica subsidiadas por el Estado, reservando espacios de cultivo en parte de los terrenos en los cuales se construye.

Por otro lado, si bien se considera posible integrar esta actividad a través de la normativa urbana, ello reviste una complejidad debido a la predominancia que en nuestros tiempos tiene la variable económica dentro del desarrollo social, pues su incorporación a la planificación urbana no sería económicamente rentable. Sin embargo, en este sentido el prisma se pierde

${ }^{6}$ DE ZEEUW et. al, 2000; 5

7 AROSEMENA, 2005; 5

8 GUERRA, 2009

${ }^{9}$ DE ZEEUW et. al., 2000 


\section{REVISTA DE \\ URBANISMO}

ISSN 0717-5051

http://revistaurbanismo.uchile.cl
Revista de Urbanismo N²9 - Diciembre de 2013 Departamento de Urbanismo - FAU - Universidad de Chile

porque no se comprende que los verdaderos beneficios de la agricultura urbana tienen que ver con aspectos relativos a lo social, a la salud, y al medioambiente, los cuales van más allá del aspecto monetario. En palabras de Graciela Ramos, tienen que ver con el bien común de nuestra sociedad, por lo tanto, el Estado debiese tomar parte en este asunto.

Por último, es preocupante constatar que la sociedad no tiene conciencia de la fragilidad del sistema alimenticio actual basado en los flujos, en el que las ciudades para abastecerse, dependen del transporte desde áreas principalmente lejanas y del suministro asociado a centros específicos de distribución de alimentos (supermercados, ferias, mercados), modelo que podría desestabilizarse y afectar el sostenimiento de nuestras ciudades. Por las condiciones geográficas de Chile, en las que no existe una red de ciudades interconectadas, sino más bien una conexión lineal entre ellas, no sería difícil que las vías de conexión se interrumpieran aislando a una o más ciudades. Por esta razón es que la agricultura urbana representa una manera de contribuir al equilibrio de las ciudades, en las que una planificación sustentable es imperativa. Esto será posible únicamente si existe un interés por parte del Estado para tomar las iniciativas existentes e incorporarlas dentro de la planificación de las ciudades. 


\section{Bibliografía}

AROSEMENA, Graciela, "Cultivar la Ciudad", en Ideas Sostenibles [en línea]. 2005, año 2, № 11, págs. 15. [Visitado abril 24, 2013] http://upcommons.upc.edu/revistes/bitstream/2099/231/1/11_cultivarciutat_Arosemena_CAST.pdf

DE ZEEUW, Henk ; Guendel, Sabine y Waibel, Hermann, "The Integration of Agriculture in Urban Policies" [en línea], en Urban Agriculture Magazine. 2000, №1, vol. 1, 6 págs. [Visitado abril 25, 2013] Disponible en http://www.ruaf.org/node/108

FERNANDEZ, José; Holmes, Felipe. Derecho Urbanístico Chileno. 3era edición, Santiago de Chile, Editorial Jurídica de Chile, 2012, 392 págs.

GUERRA, Constanza, Evaluación ambiental de la tierra vacante del área metropolitana del gran Santiago y su relación con los grupos socioeconómicos de la población y el valor del suelo [en línea], tesis de Pregrado de Geografía, Santiago de Chile, 2009, 83 págs. [Visitado mayo 12, 2013] Disponible en http://www.tesis.uchile.cl/handle/2250/100104

I. MUNICIPALIDAD DE LA PINTANA. Proyecto Plan Regulador Comunal [en línea]. [Visitado junio 1, 2013] Disponible

http://www.pintana.cl/transparencia/index.php?option=com_content\&task=view\&id=78\&Itemid $=121$

I. MUNICIPALIDAD DE LA REINA. Ordenanza Plan Regulador [en línea]. Documento en línea: 2001, 124 págs. [Visitado junio 2, 2013] Disponible en http://www.lareina.cl/la_reina/plan_regulador.php

I. MUNICIPALIDAD DE LA REINA. Plano de Zonificación [en línea]. Documento en línea: 2010, 1 pág. [Visitado junio 2, 2013] Disponible en http://www.lareina.cl/la reina/doc plan regulador/PRCLR 012010 ZONIFICACION.pdf

I. MUNICIPALIDAD DE LA REINA. Reseña histórica [en línea]. [Visitado junio 1, 2013] Disponible en http://www.lareina.cl/la_reina/comuna_resena.php

I. MUNICIPALIDAD DE LA REINA. Unidad de Medioambiente [en línea]. [Visitado mayo 30, 2013] Disponible en http://www.lareina.cl/medio_ambiente/agricultura.php

MINISTERIO DE VIVIENDA Y URBANISMO. Plan Regulador Metropolitano de Santiago [en línea]. Documento en línea: 2010. [Visitado abril 9, 2013] Disponible en http://www.seremi13minvu.cl/incjs/download.aspx?glb cod nodo $=20080710120043 \&$ hdd no m archivo=Ordenanza PRMS Actualizaci\%C3\%B3n Mayo\%20201001.pdf

ORGANISATION FOR ECONOMIC CO-OPERATION AND DEVELOPMENT (OCDE). National Urban Policy Reviews - The Case of Chile [en línea]. Documento en línea: 2013, 203 págs. [Visitado junio 11, 2013] Disponible

http://search.oecd.org/officialdocuments/publicdisplaydocumentpdf/?cote=GOV/TDPC/URB\%28 2012\%2913\&docLanguage $=$ En

PAZ, René, "Primer estudio de huertos urbanos contabiliza 39 en Santiago" [en línea]. En La Tercera. 2012. [Visitado mayo 9, 2013] Disponible en http://www.latercera.com/noticia/santiago/2012/12/1731497125-9-primer-estudio-de-huertos-urbanos-contabiliza-39-en-santiago.shtml

WWF. Sustainable Farming [en línea]. [Visitado abril 4, 2013] Disponible en http://wwf.panda.org/what we do/footprint/agriculture/ 